\title{
Spherical contours, IR divergences and the geometry of Feynman parameter integrands at one loop
}

\author{
Akshay Yelleshpur Srikant \\ Department of Physics, Princeton University, \\ Washington Road, Princeton, NJ 08540, U.S.A. \\ E-mail: ysakshay@princeton.edu
}

ABSTRACT: Spherical contours introduced in [1] translate the concept of "discontinuity across a branch cut" to Feynman parameter space. In this paper, we further explore spherical contours and connect them to the computation of leading IR divergences of 1 loop graphs directly in Feynman parameter space. These spherical contours can be used to develop a Feynman parameter space analog of "Leading Singularities" of loop integrands which allows us to develop a method of determining Feynman parameter integrands without the need to sum over Feynman diagrams in momentum space. Finally, we explore some interesting features of Feynman parameter integrands in $\mathcal{N}=4$ SYM.

KeYwords: 1/N Expansion, Scattering Amplitudes

ArXiv EPrint: 1907.05429 


\section{Contents}

1 Introduction $\quad 1$

2 Feynman parametrization revisited 2

3 1-loop IR divergences 4

3.1 Composite residues in momentum space $\quad 7$

3.2 Composite residues in Feynman parameter space 8

$\begin{array}{lr}3.3 \text { Proof for general one-loop integrals } & 9\end{array}$

3.4 A basis for IR finite integrals in Feynman parameter space 11

4 Algebraic aspects of spherical residues $\quad 12$

4.1 Properties of Feynman integrals coming from loop integrals 14

$\begin{array}{lll}4.2 & \text { Spherical contours meet IR divergences } & 15\end{array}$

5 Constructing integrands using spherical residues $\quad 16$

$\begin{array}{lll}5.15 \text { point integrands } & 16\end{array}$

$\begin{array}{ll}5.26 \text { point integrands } & 17\end{array}$

6 Feynman parametrization in planar $\mathcal{N}=4$ SYM 18

$\begin{array}{lll}7 & \text { Outlook } & 19\end{array}$

$\begin{array}{ll}\text { A Cuts of Feynman integrals } & 20\end{array}$

$\begin{array}{ll}\text { B Spherical contour with a quadratic numerator } & 21\end{array}$

C Leading singularities at 6 points 22

D Feynman parametrizing the MHV planar 1-loop integrand 23

\section{Introduction}

A connection between the singularity structure of one-loop integrals and the projective geometry of their associated Feynman parameter integrand was established in [1]. One of the central results of this paper was the introduction of a new kind of residue in Feynman parameter space, associated with "spherical contours" which were operations involving only the one-loop integrand. These capture information about discontinuities of the integrals across various branch cuts. It was shown that this calculus based operation also has an algebraic interpretation. The purpose of this paper is to provide some additional details 
on this algebraic interpretation and also explore IR divergent integrals as the authors of [1] largely focused on finite integrals.

The structure of the paper is as follows. We begin by discussing some preliminaries of Feynman parametrization and setting the notation for the rest of the paper. In section 3, we investigate IR divergent integrals in Feynman parameter space. We motivate and develop a new kind of "residue" operation which computes the leading IR divergence of one loop amplitudes. We use the simple example of a scalar $n$-gon integral to demonstrate the procedure. We then use the embedding space formalism [2] to consider general one loop integrals and demonstrate that this procedure correctly reproduces the leading IR divergences. Section 4 involves a discussion of the algebraic structure of spherical residues. In particular, we provide expressions for the new numerators of spherical residues of integrands. We then prove a few essential properties of spherical residues. In section 5, we outline a method to construct one-loop integrands using spherical residues. Finally, we conclude by examining some appealing features of Feynman parameter integrands in $\mathcal{N}=4 \mathrm{SYM}$ in 6 .

\section{Feynman parametrization revisited}

Although Feynman parametrization is a familiar trick, let us begin by discussing it in a more geometric way. This will highlight some of the features of Feynman parameter integrals which are important for the rest of the paper. Consider the scalar, one-loop integrals of the form ( $\mu^{2}$ is the mass scale introduced in dimensional regularization)

$$
I_{n}=\left(\mu^{2}\right)^{\nu-L D / 2} \int \prod_{k=1}^{L} \frac{d^{D} \ell_{k}}{i \pi^{D / 2}} \prod_{j=1}^{n} \frac{1}{\left(-q_{j}^{2}+m_{j}^{2}\right)^{\nu_{j}}} \quad \nu=\sum_{j=1}^{n} \nu_{j} .
$$

Here each $q_{j}$ is a linear combination of the external momenta $p_{k}$ and the loop momenta $\ell_{k}$. A straightforward Feynman parametrization yields (see [3-5])

$$
I_{n}=\left(\mu^{2}\right)^{\nu-L D / 2} \frac{\Gamma(\nu-L D / 2)}{\prod_{j=1}^{n} \Gamma\left(\nu_{j}\right)} \int_{0}^{\infty} d^{n} x \delta\left(1-\sum_{i} x_{i}\right) \prod_{j=1}^{n} x_{j}^{\nu_{j}-1} \frac{\mathcal{U}^{\nu-(L+1) D / 2}}{\mathcal{F}^{\nu-L D / 2}},
$$

where $\mathcal{U}$ and $\mathcal{F}$ are functions of the Feynman parameters $x_{j}$ and the external momenta and depend on the particular integral being evaluated. They are connected to the propagators appearing in eq. (2.1) via the polynomial

$$
\sum_{j=1}^{n} x_{j}\left(-q_{j}^{2}+m_{j}^{2}\right)=-\sum_{r, s=1}^{L} \ell_{r}^{\mu} M_{r, s} \ell_{s \mu}+2 \sum_{r=1}^{L} l_{r}^{\mu} Q_{r \mu}+J
$$

where $J$ contains all the terms independent of the loop momenta. Then,

$$
\mathcal{U}=\operatorname{det} M \quad \mathcal{F}=\operatorname{det} M\left(J+Q \cdot M^{-1} \cdot Q\right) .
$$

$\mathcal{U}$ and $\mathcal{F}$ are called the Symanzik polynomials. It will be of interest to note that at one-loop, $\mathcal{U}$ and $\mathcal{F}$ are homogeneous linear and quadratic polynomials respectively. The Symanzik 
polynomials can also be calculated efficiently by using graphical rules. For more details on these rules, we refer the reader to [3].

It is illuminating to consider an alternate derivation of this result. Let us first introduce Schwinger parameters $\alpha_{i}$

$$
\frac{1}{\left(-q_{i}^{2}+m_{i}^{2}\right)^{\nu_{i}}}=\int_{0}^{\infty} d \alpha_{i} e^{-\alpha_{i}\left(-q_{i}^{2}+m_{i}^{2}\right)^{\nu_{i}}} .
$$

Inserting this in eq. (2.1), we can perform the Gaussian integrals over all the loop momenta. The result is

$$
I_{n}=\left(\mu^{2}\right)^{\nu-L D / 2} \frac{i^{-\nu-1} \pi^{2}}{\prod_{j=1}^{n} \Gamma\left(\nu_{j}\right)} \int_{0}^{\infty} d \alpha_{1} \ldots d \alpha_{n} \prod_{j=1}^{n} \alpha_{j}^{\nu_{j}-1} \frac{1}{U^{D / 2}} e^{i \frac{F}{U}-i \sum_{j} m_{j}^{2} \alpha_{j}},
$$

where $U$ and $F$ are polynomials in the $\alpha_{i}$. They are homogeneous and like the Symanzik polynomials $\mathcal{U}$ and $\mathcal{F}$, linear and quadratic respectively. For more details, see $[3,6]$.

We can now introduce new variables via $\alpha_{i}=\eta x_{i}$. Since there are $n+1$ new variables, we must impose a constraint on the $x_{i}$ which we take to be $\sum_{i \in S} x_{i}=1$, where $S \subset$ $\{1, \ldots n\}$. This changes eq. (2.4) to

$$
I_{n}=\left(\mu^{2}\right)^{\nu-L D / 2} \frac{i^{-\nu-1} \pi^{2}}{\prod_{j=1}^{n} \Gamma\left(\nu_{j}\right)} \int_{0}^{\infty} d X d \eta \eta^{n-1}\left(\eta^{\nu-n-2} \prod_{j=1}^{n} x_{j}^{\nu_{j}-1} \frac{1}{U^{D / 2}} e^{i \eta\left(\frac{F}{U}-i \sum_{j} m_{j}^{2} x_{j}\right)}\right),
$$

where $d X=d x_{1} \ldots d x_{n} \delta\left(1-\sum_{i \in S} x_{i}\right)$. This result is called the Cheng-Wu theorem [7]. In particular, this implies that we could set any one of the Feynman parameters $x_{i}$ to 1 . The vector $X=\left(x_{1}, \ldots, x_{n}\right)$ can be thought of as a point in projective space and the measure $d x_{1} \ldots d x_{n} \delta\left(1-\sum_{i \in S} x_{i}\right)$ can be better written as

$$
\left\langle X d^{n-1} X\right\rangle \equiv \epsilon_{\mu_{1} \ldots \mu_{n}} X^{\mu_{1}} d X^{\mu_{2}} \ldots d X^{\mu_{n}} .
$$

The textbook result of Feynman parametrization eq. (2.2) is obtained by setting $S=$ $\{1, \ldots n\}$. For the rest of the paper, we will only work with integrals with the factors $\nu_{i}=1$ and write all the Feynman parameter integrals in a projective manner as shown below (the factor of $\left(\mu^{2}\right)^{n-L D / 2}$ is omitted).

$$
I_{n}=\Gamma(n-L D / 2) \int \frac{\left\langle X d^{n-1} X\right\rangle \mathcal{U}^{n-(L+1) D / 2}}{\mathcal{F}^{n-L D / 2}} .
$$

The homogeneity properties of $\mathcal{U}$ and $\mathcal{F}$ are essential in making the integrals projectively well defined.

Throughout this paper we will use three kinds of variables to describe the external momenta-dual momenta, momentum twistors [8] and embedding space momenta [2]. Dual momenta $y_{i}^{\mu}$ are defined by

$$
p_{i}^{\mu}=y_{i}^{\mu}-y_{i-1}^{\mu} \quad y_{i j}^{\mu} \equiv y_{i}^{\mu}-y_{j}^{\mu} .
$$

In the rest of the paper, we focus on one-loop integrals and associate a dual variable $y$ with the loop momentum. Momentum twistors, $Z_{i}$, introduced in [8], are convenient to 
describe null momenta in $4 \mathrm{D}$. They are defined by associating a line $Z_{i-1} Z_{i}$ with each $y_{i}$. The scalar $y_{i j}^{2}$ is related to the $\mathrm{SL}(4, R)$ invariant $\langle i-1 i j-1 j\rangle \equiv \epsilon_{A B C D} Z_{i-1}^{A} Z_{i}^{B} Z_{j-1}^{C} Z_{j}^{D}$. Each loop momentum variable is associated to a line $A B$ in twistor space which is to be integrated over using the measure $\left\langle A B d^{2} A\right\rangle\left\langle A B d^{2} B\right\rangle$. For more details, see [9]

A vector $y^{\mu}$ in D-dimensional Minkowski space is mapped to a null vector $Y^{M}=$ $\left(1, y^{2}, y^{\mu}\right)$ in embedding space. Here, we have specified the components in light-cone coordinates, i.e. $Y^{+}=1, Y^{-}=y^{2}$ and $Y^{\mu}=y^{\mu}$. The metric is $g_{+-}=g_{-+}=-1 / 2$ and $g_{\mu \nu}=\eta_{\mu \nu}$ with all other entries zero. The invariants $y_{i j}^{2}=-2 Y_{i} . Y_{j}$. In particular, for null momenta, we have $Y_{i} \cdot Y_{i+1}=0$. The integral eq. (2.1) can be written as

$$
I_{n}=\left(\mu^{2}\right)^{\nu-L D / 2} \int \frac{\left[d^{4} Y\right]}{\left(Y . Y_{1}\right)^{\nu_{1}} \ldots\left(Y . Y_{n}\right)^{\nu_{n}}} .
$$

The measure $\left[d^{4} Y\right]=\frac{d^{6} Y \delta(Y . Y)}{\operatorname{Vol}(\mathrm{GL}(1))}$. For more details, see $[1,2]$.

For the particular case of planar one-loop integrals, simple expressions are available for the Symanzik polynomials. While $\mathcal{U}$ depends on the details of the numerator, $\mathcal{F}$ depends only on the pole structure.

$$
\mathcal{F}=\sum_{i<j} x_{i} x_{j} y_{i j}^{2}=X . Q . X
$$

where $Q_{i j}$ can be expressed in any of the three equivalent forms $y_{i j}^{2},\langle i-1 i j-1 j\rangle$ or $Y_{i} . Y_{j}$.

\section{1-loop IR divergences}

The infrared (IR) structure of gauge theories has been the subject of a great amount of study. Long range forces which lead to early and late time interactions in a scattering process make the very definition of an S-Matrix difficult. For recent work on these issues, see $[10,11]$. One of the main reasons for these difficulties is the contribution of IR divergent loop integrals to scattering amplitudes. These integrals are usually dealt with via dimensional regularization. In $D=4-2 \epsilon$ dimensions, at one-loop, soft and collinear divergences appear as $\frac{1}{\epsilon}$ poles and overlapping soft-collinear divergences lead to $\frac{1}{\epsilon^{2}}$ poles. At L-loops, the leading divergence is $\frac{1}{\epsilon^{2 L}}$.

The structure of these divergences is well understood in gauge theories. It has been shown that they have a universal (independent of the number of external particles) structure which is captured by the factorization formula

$$
\mathcal{M}_{n}=S\left(\left\{k_{i}\right\}, \mu, \epsilon\right) \times \prod_{i=1}^{n} J_{i}\left(k_{i}, \mu, \epsilon\right) \times h_{n}\left(\left\{k_{i}\right\}, \mu\right) .
$$

Here $\mathcal{M}_{n}$ is the full $n$-particle amplitude divided by the corresponding tree amplitude, $\mu$ is the factorization scale (an arbitrary scale chosen to separate soft and hard momenta) and $\epsilon$ is the dimensional regularization parameter. In writing this, we have followed the notation in [12]. $h_{n}$ is a finite function corresponding to hard scattering, $S$ is a color dependant function which encodes the soft singularities and $J_{i}$ are functions encoding the collinear 
singularities along the $k_{i}$ directions. In general, these are all complicated matrices in color and spin space. For a review of factorization properties and the structure of IR divergences in QCD and other gauge theories, see [13, 14].

The factorization in eq. (3.1) has introduced an arbitrary scale $\mu$ which distinguishes between hard and soft momenta. This scale is unphysical and amplitudes cannot depend on it. Consequently, $\mathcal{M}_{n}$ satisfies a renormalization group equation

$$
\mu \frac{d}{d \mu} S\left(\left\{k_{i}\right\}, \mu, \epsilon\right)=-\Gamma S\left(\left\{k_{i}\right\}, \mu, \epsilon\right)
$$

with $\Gamma$ being the anomalous dimensions matrix. It encodes the structure of IR divergences in the theory. For more details, we refer the reader to the review [15].

In planar theories and conformal theories like $\mathcal{N}=4$, the all-loop solution to the RG equations is easy to obtain. Surprisingly, the full four point amplitude in $\mathcal{M}_{4}$ is completely fixed by the structure of IR divergences. It can be written as [16]

$$
\mathcal{M}_{4}=\exp \left[-\frac{1}{8} \sum_{l=1}^{\infty} a^{l}\left(\frac{\gamma_{K}^{(l)}}{(l \epsilon)^{2}}+\frac{2 \mathcal{G}_{0}^{(l)}}{l \epsilon}\right) \sum_{i=1}^{4}\left(\frac{\mu^{2}}{-s_{i, i+1}}\right)^{l \epsilon}\right]
$$

where $a$ is related to the coupling and the number of colours, $l$ is the number of loops and $\gamma_{K}^{(l)}$ and $\mathcal{G}_{0}^{(l)}$ are constants that are not fixed by the RG equations. The cusp anomalous dimension is $\Gamma_{\mathcal{M}_{n}}=\sum_{l} a^{l} \gamma_{K}^{(l)}$. At one-loop, it is simply the coefficient of $\frac{1}{\epsilon^{2}}$. At higher points, while the structure of the IR divergences doesn't change, the full amplitude is different from 3.3 and involves remainder functions. Techniques from integrability provide all order results for $\Gamma_{\mathcal{M}_{n}}$ [17] which has been tested via perturbative computations extensively [18-21]. The IR divergences are related to the UV divergences of lightlike cusps of Wilson loops $[22,23]$. The UV divergences of these Wilson loops are controlled by the same anomalous dimension, justifying the name "cusp" anomalous dimension.

The focus of this paper is on understanding the origin of IR divergences in Feynman parameter space and in developing a method to compute the leading IR divergent behaviour directly in Feynman parameter space (in 4 spacetime dimensions). The first step is to gain an understanding of one-loop integrals. The complete structure of IR divergences in a theory depends on the particle content of the theory. We will not delve into these issues here and will focus solely on the properties of individual integrals. The next few paragraphs serve to provide intuition for analyzing IR divergences in Feynman parameter space.

It is well known [14] that IR divergences arise when the loop momentum $\ell$ becomes collinear with an external massless momentum $p_{i}$, i.e. $\ell . p_{i} \rightarrow 0$ (soft) or when it becomes collinear to two consecutive null external momenta $\ell \cdot p_{i-1}=\ell . p_{i}=0$. Soft-collinear divergences arise when both these conditions are satisfied simultaneously. Let us consider the concrete example of a 4D massless box integral (figure 1) in momentum space and the corresponding Feynman parameter integral (in dimensional regularization with $D=4-2 \epsilon$ ).

$$
\begin{aligned}
I_{4} & =\int \frac{d^{D} \ell}{i \pi^{D / 2}} \frac{1}{\ell^{2}\left(\ell-p_{2}\right)^{2}\left(\ell-p_{2}-p_{3}\right)^{2}\left(\ell+p_{1}\right)^{2}} \\
& =\int \prod_{i=1}^{4} d x_{i} \Gamma(2+\epsilon) \frac{\delta\left(x_{1}+x_{2}+x_{3}+x_{4}-1\right)}{\left(x_{1} x_{3} s+x_{2} x_{4} u\right)^{2+\epsilon}},
\end{aligned}
$$




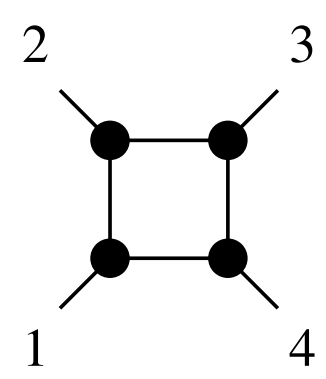

Figure 1. Box integral.

where $s=\left(p_{1}+p_{2}\right)^{2}$ and $u=\left(p_{1}+p_{4}\right)^{2}$. This integral is, of course, well known and has been evaluated in dimensional regularization in $D=4-2 \epsilon$ dimensions (re-introducing the factors of $\left.\mu^{2}\right)[24,25]$ to

$$
I_{4}=\frac{\Gamma(1+\epsilon) \Gamma^{2}(1-\epsilon)}{\Gamma(1-2 \epsilon) s u}\left(\frac{2}{\epsilon^{2}}\left[\left(-\mu^{-2} s\right)^{-\epsilon}+\left(-\mu^{-2} u\right)^{-\epsilon}\right]-\log ^{2} \frac{s}{u}-\pi^{2}\right)+\mathcal{O}(\epsilon)
$$

The presence of the $\frac{1}{\epsilon^{2}}$ terms indicated the presence of IR divergences. A more transparent analysis using a massive regulator instead of dimensional regularization reveals that the divergence coming from the regions $\ell^{2}=\left(\ell-p_{2}\right)^{2}=\left(\ell+p_{1}\right)^{2}=0$ is of the form $\frac{1}{s u}\left(\log ^{2} \frac{m^{2}}{s}+\right.$ $\left.\log ^{2} \frac{m^{2}}{u}\right)$. We refer the reader to section 7 in [26].

We can thus precisely characterize the leading IR divergent region in momentum space as being associated with three propagators going on-shell. A similar characterization in Feynman parameter space should involve the Feynman parameters corresponding to these three propagators, $x_{1}, x_{2}$ and $x_{3}$. To motivate such a characterization, recall the definition of the Schwinger parameter $\alpha$

$$
\frac{1}{p^{2}}=\int_{0}^{\infty} d \alpha e^{-\alpha p^{2}}
$$

Near the upper limit of the integral, i.e. for large $\alpha$, a configuration with $p^{2} \approx 0$ would be the most relevant. We might hope that the large $\alpha$ limit probes soft momenta. Since Feynman parameters are related to Schwinger parameters by $x_{i}=\frac{\alpha_{i}}{\sum_{i} \alpha_{i}}$, the limit $\alpha_{i} \rightarrow \infty$ corresponds to $x_{i} \rightarrow 1$ non-projectively or $x_{i} \rightarrow \infty$ projectively.

Furthermore, we can manipulate $I_{4}$ to understand the relationship between the consecutive massless legs and IR divergences. We can use Lorentz invariance to transform to a frame in which $p_{1}^{\mu}=(1,0,0,0)$ and $p_{2}^{\mu}=(0,1,0,0)$. Here, the components have been specified in light-cone frame as $\left(p_{i}^{+}, p_{i}^{-}, p_{i}^{1}, p_{i}^{2}\right)$. Hence $p_{1}^{2}=p_{2}^{2}=0$ is automatic. If we work in the soft region where $\ell^{2} \approx 0$, we can write $\ell . p_{1} \approx l_{+}$and $\ell . p_{2} \approx l_{-}$. In this case, we can schematically write the integral as follows

$$
I_{4} \approx \int \frac{1}{2 p_{2} \cdot p_{3}} \frac{d^{2} \ell_{\perp}}{\left(\ell_{+} \ell_{-}-\ell_{\perp}^{2}\right)} \frac{d \ell_{-}}{\ell_{-}} \frac{d \ell_{+}}{\ell_{+}}
$$


(where we have set $\epsilon=0){ }^{1}$ The soft collinear region is the region in which all three propagators go on shell. $\ell_{+} \approx 0, \ell_{-} \approx 0$ and $\ell_{\perp}^{2} \approx \ell_{+} \ell_{-}$. In combination with the intuition in the previous paragraph, this suggests that the region in Feynman parameter space which gives rise to the divergence is $x_{2} \approx x_{1} x_{3}$ and $x_{2} \rightarrow \infty$. In this region, we should be able to observe a $\log ^{2}$ divergence and calculate its coefficient. This should be equal to the coefficient of $\frac{1}{\epsilon^{2}}$ in dimensional regularization which is the cusp anomalous dimension $\Gamma$. Note that this is purely a conjecture at this point and in the next section, we will demonstrate that this region in Feynman parameter space indeed captures the essential information about the IR divergent region and can be used to calculate $\Gamma$.

\subsection{Composite residues in momentum space}

Let us begin by understanding the calculation of $\Gamma$ directly in momentum space as a composite residue on the poles corresponding to three propagators going on shell. The idea of composite residues was first introduced in [27, 28]. Here, we compute this for a scalar n-gon.

$$
\begin{aligned}
I_{n} & =\int \frac{d^{4} l}{l^{2}\left(l-p_{2}\right)^{2} \ldots\left(l+p_{1}\right)^{2}} \\
& =\int \frac{d^{4} y}{\left(y-y_{1}\right)^{2}\left(y-y_{2}\right)^{2} \ldots\left(y-y_{n}\right)^{2}}
\end{aligned}
$$

where

$$
p_{i}=y_{i}-y_{i-1} \quad y=l+x_{1} \quad\left(l-\sum_{i=2}^{k} p_{i}\right)=l+y_{1}-y_{k} .
$$

We want to calculate the residue associated with the loop momentum $\ell$ being collinear to two consecutive null external momenta, i.e. $\ell \cdot p_{i-1}=\ell \cdot p_{i}=0$. In terms of the dual momenta $y_{i}$, this is equivalent to $\left(y-y_{i}\right)^{2}=\left(y-y_{i-1}\right)^{2}=\left(y-y_{i+1}\right)^{2}=0$. To calculate this residue, we first parametrize $y$ on the cut $\left(y-y_{i}\right)^{2}=0$ by introducing spinor helicity variables, (see [25] for a review)

$$
y^{\mu}=y_{i}^{\mu}+\sigma_{\alpha \dot{\alpha}}^{\mu} \lambda^{\alpha} \tilde{\lambda}^{\dot{\alpha}} \quad y_{i}^{\mu}-y_{i-1}^{\mu}=\sigma_{\alpha \dot{\alpha}}^{\mu} \lambda_{i}^{\alpha} \tilde{\lambda}_{i}^{\dot{\alpha}} .
$$

From this, it follows that

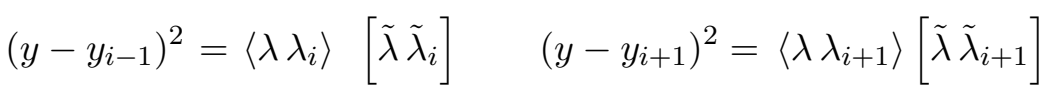

$$
\begin{aligned}
& \left(y-y_{k}\right)^{2}=\left(y_{i}-y_{k}\right)^{2}+2\left(y_{i}-y_{k}\right)^{\mu} \sigma_{\mu \alpha \dot{\alpha}}\left(\lambda^{\alpha} \tilde{\lambda}^{\dot{\alpha}}\right),
\end{aligned}
$$

where $\langle a b\rangle=\epsilon_{\alpha \beta} a^{\alpha} b^{\beta}$ and $[\tilde{a} \tilde{b}]=\epsilon_{\dot{\alpha} \dot{\beta}} \tilde{a}^{\dot{\alpha}} \tilde{b}^{\dot{\beta}}$. For convenience, we expand $\lambda$ in a basis consisting of $\lambda_{i}$ and $\lambda_{i+1}$ (with a similar expansion for $\tilde{\lambda}$ ).

$$
\lambda=\beta \lambda_{i}+\gamma \lambda_{i+1} \quad \tilde{\lambda}=\sigma \tilde{\lambda}_{i}+\rho \tilde{\lambda}_{i+1} .
$$

\footnotetext{
${ }^{1}$ Formally, the integral is ill defined and doesn't exist. However, it suffices to illustrate the point schematically. In the rest of the paper, we will be interested in computing residues which are well defined and do not require a regulator.
} 
In terms of these variables, the measure on the cut $\left(y-y_{i}\right)^{2}=0$ is

$$
\int d^{4} y \delta\left(\left(y-y_{i}\right)^{2}\right)=\int \frac{d^{2} \lambda d^{2} \tilde{\lambda}}{\operatorname{Vol} \operatorname{GL}(1)}=\int d \gamma d \rho d \sigma\left\langle\lambda_{i} \lambda_{i+1}\right\rangle\left[\lambda_{i} \lambda_{i+1}\right] .
$$

We have used the GL(1) to fix $\beta=1$. By introducing spinor helicity variables, we are already on the cut $\left(y-y_{i}\right)^{2}=0$. This residue can now be written as

$$
\operatorname{Res}_{\left(y-y_{i}\right)^{2}=0} I_{n}=\int \frac{d \gamma}{\gamma} \frac{d \rho}{\rho} \frac{d \sigma}{\sigma} \frac{1}{\left\langle\lambda_{i} \lambda_{i+1}\right\rangle\left[\lambda_{i} \lambda_{i+1}\right] \prod_{k \neq\{i-1, i, i+1\}}\left(\left(y_{i}-y_{k}\right)^{2}+2\left(y_{i}-y_{k}\right)^{\mu} \sigma_{\mu \alpha \dot{\alpha}} \lambda^{\alpha} \tilde{\lambda}^{\dot{\alpha}}\right)} .
$$

On this cut, we can now fully localize the loop momentum $y_{i}$ by taking the residue of the poles $\gamma=\rho=\sigma=0$, even though we have cut only three propagators. This is an example of a composite residue. Recalling that $\left\langle\lambda_{i} \lambda_{i+1}\right\rangle\left[\lambda_{i} \lambda_{i+1}\right]=2\left(y_{i}-y_{i-1}\right) \cdot\left(y_{i+1}-y_{i}\right)=y_{i-1 i+1}^{2}$, the co-efficient of the IR divergence can be written as

$$
\Gamma_{I_{n}}=\oint_{\gamma=\rho=\sigma=0} \operatorname{Res}_{\left(y-y_{i}\right)^{2}=0} I_{n}=\frac{1}{2 y_{i-1, i+1}^{2} \prod_{k \neq\{i-1, i, i+1\}} y_{i, k}^{2}} .
$$

This can be compared to the full expression for the amplitudes given in [24, 29].

\subsection{Composite residues in Feynman parameter space}

We will now demonstrate that the coefficient of the $\log ^{2}$ divergence, as obtained in eq. (3.6) can also be obtained directly in Feynman parameter space. As suggested above, the IR divergences in Feynman parameter space are associated to a triplet of consecutive Feynman parameters $\left(x_{i-1}, x_{i}, x_{i+1}\right)$ and come from the region where $x_{i}$ is large and $x_{i-1} x_{i+1}$ scales as $x_{i}$. We will evaluate the integral eq. (3.5) in this limit and find that the result is proportional to $\Gamma_{I_{n}}$.

We being by writing (3.5) as a projective integral in Feynman parameter space.

$$
I_{n}=\Gamma(n-2) \int \frac{\left\langle X d^{n-1} X\right\rangle \mathcal{U}^{n-4}}{\mathcal{F}^{n-2}}
$$

with the Symanzik polynomials

$$
\mathcal{U}=\sum_{i} x_{i} \quad \mathcal{F}=\sum_{i<j} x_{i} x_{j} y_{i j}^{2}
$$

Let us introduce new variables $(\rho, \tau)$,

$$
x_{i-1}=\sqrt{x_{i}} \rho e^{\tau} \quad x_{i+1}=\sqrt{x_{i}} \rho e^{-\tau} .
$$

This change of variables ensures that we have the required scaling, $x_{i-1} x_{i+1}=\rho^{2} x_{i}$ of the relevant Feynman parameters. In the limit of the limit of large $x_{i}$, the Symanzik polynomials reduce to

$$
\begin{aligned}
& \mathcal{U}=x_{i}+\mathcal{O}\left(\sqrt{x_{i}}\right) \\
& \mathcal{F}=x_{i}\left(y_{i-1 i+1}^{2} \rho^{2}+\sum_{j \neq\{i-1, i, i+1\}} y_{i j}^{2} x_{j}\right)+\mathcal{O}\left(\sqrt{x_{i}}\right) .
\end{aligned}
$$


Note that the quadric has factorized in this limit. This guarantees that the resulting integral over the remaining $(n-3)$ Feynman parameters (recall that the integral is projective and requires only $(n-1)$ integrations) is now rational.

$$
\begin{aligned}
I_{n} & \approx \int\left(\frac{\prod_{k \neq i-1, i+1} d x_{k}}{\operatorname{VolGL}(1)}\right) 2 \rho x_{i} d \rho d \tau \frac{\left(x_{i}^{n-4}\right) \Gamma(n-2)}{x_{i}^{n-2}\left(y_{i-1 i+1}^{2} \rho^{2}+\sum_{j \neq\{i-1, i, i+1\}} y_{i j}^{2} x_{j}\right)^{n-2}} \\
& =\Gamma(n-2) \int \frac{d x_{i}}{x_{i}} d \tau \int \frac{\prod_{j \neq\{i-1, i, i+1\}} d x_{j}}{\operatorname{VolGL}(1)} \frac{2 \rho d \rho}{\left(y_{i-1 i+1}^{2} \rho^{2}+\sum_{j \neq\{i-1, i, i+1\}} y_{i j}^{2} x_{j}\right)^{n-2}},
\end{aligned}
$$

where we've explicitly written out a factor of Vol GL(1) to indicate that the $x_{i}$ are projective. The divergent factor is

$$
\int \frac{d x_{i}}{x_{i}} d \tau=\frac{1}{2} \int d \log x_{i} d \log \frac{x_{i+1}}{x_{i-1}} .
$$

After fixing the GL(1) redundancy by setting one of the $x_{j}=1$, the remaining integral is rational and can be easily evaluated.

$$
2 \Gamma(n-2) \int \frac{\prod_{j \neq\{i-1, i, i+1\}} d x_{j}}{\operatorname{VolGL}(1)} \rho d \rho \frac{1}{\left(\rho^{2}+\sum_{j \neq i-1, i, i+1} x_{j}\right)^{n-2}}=1 .
$$

With this,

$$
I_{n} \approx \frac{1}{2} \int d \log x_{i} d \log \left(\frac{x_{i+1}}{x_{i-1}}\right) \frac{1}{y_{i-1, i+1}^{2} \prod_{j \neq\{i-1, i, i+1\}} y_{i j}^{2}}
$$

where the $\approx$ sign indicates that this result is true only in the above limit. We see that there is a $\log ^{2}$ divergence and its coefficient is identical to $\Gamma_{I_{n}}$ computed directly as a composite residue in eq. (3.6), i.e.

$$
\Gamma_{I_{n}}=\frac{1}{2} \frac{1}{y_{i-1 i+1}^{2} \prod_{j \neq\{i-1, i, i+1\}} y_{i j}^{2}} .
$$

\subsection{Proof for general one-loop integrals}

In the previous section, we showed that the scaling procedure correctly isolates the leading IR divergence in Feynman parameter space. However, the proof presented there is valid only for scalar $n$-gon integrals. This is due to the specific form of the Symanzik polynomials in eq. (3.8). In this section, we will generalize the above results to include cases with arbitrary tensor numerators. We will show that the coefficient of the IR divergence obtained by computing a composite residue involving three propagators is the same as the one obtained in Feynman parameter space using the procedure outlined in section 3.2.

For the purposes of generalizing the proof, it is easiest to work in embedding space. We will use the formalism and notation of section[4] of [1]. A generic one-loop integral with a tensor numerator has the form

$$
I_{n}=\int \frac{T\left[Y^{n-4}\right]\left[d^{4} Y\right]}{\left(Y . Y_{1}\right) \ldots\left(Y . Y_{n}\right)}
$$

where $T\left[Y^{n-4}\right]=T_{i_{1} \ldots i_{n}} Y^{i_{1}} \ldots Y^{i_{n}}$ is a tensor of rank $n-4$. The measure $\left[d^{4} Y\right]=\frac{d^{6} Y \delta(Y . Y)}{\operatorname{Vol}(\operatorname{GL}(1))}$. 
To calculate the coefficient of the IR divergence, we follow the same procedure as in section 3.1. We calculate the residue on the cut $Y . Y_{1}=Y . Y_{2}=Y . Y_{3}=0$. Since the denominator is the same as in eq. (3.7), it is easy to see that the same computation goes through. The end result is,

$$
\Gamma_{I_{n}}=\frac{T\left[Y_{2}^{n-4}\right]}{2\left(Y_{1} \cdot Y_{3}\right) \prod_{k \neq\{1,2,3\}} Y_{2} \cdot Y_{k}} .
$$

We will now show that the same result can be obtained in Feynman parameter space by scaling the parameters as mentioned before. We begin by Feynman parametrizing the integral in (3.10),

$$
I_{n}=\int \frac{T\left[Y^{n-4}\right]\left[d^{4} Y\right]\left\langle X d^{n-1} X\right\rangle}{(Y . W)^{n}}
$$

where $W=\sum_{i} x_{i} Y_{i}$. To do the integral over $Y$, we note that each factor of $Y$ can be exchanged for $\frac{d}{d W}$ to get

$$
\begin{aligned}
I_{n} & =\frac{6(-1)^{n-4}}{(n-1) !} T\left[\left(\frac{d}{d W}\right)^{n-4}\right] \int \frac{\left[d^{4} Y\right]\left\langle X d^{n-1} X\right\rangle}{(Y . W)^{4}} \\
& =T\left[\left(\frac{d}{d W}\right)^{n-4}\right] \int \frac{\left\langle X d^{n-1} X\right\rangle}{(W . W)^{2}},
\end{aligned}
$$

where $T\left[\left(\frac{d}{d W}\right)^{n-4}\right]=T_{i_{1} \ldots i_{n-4}} \frac{d}{d W^{i_{1}}} \ldots \frac{d}{d W^{i_{n-4}}}$ and we have used

$$
\int \frac{\left[d^{4} Y\right]}{(W . Y)^{4}}=\frac{1}{(W . W)^{2}}
$$

To compare with (3.11), we set $x_{1}=\sqrt{x_{2}} \rho e^{\tau}, x_{3}=\sqrt{x_{2}} \rho e^{-\tau}$ and take the limit of large $x_{2}$. Once again we have $W \approx x_{2} Y_{2}$ and $W \cdot W \approx x_{2}\left(\rho^{2} Y_{1} \cdot Y_{3}+\sum_{i \neq 1,2,3} x_{i} Y_{i} \cdot Y_{2}\right)$. In the large $x_{2}$ limit, only the term $T\left[W^{n-4}\right]=x_{2}^{n-4} T\left[Y_{2}^{n-4}\right]$ contributes and

$$
I_{n} \approx \int \frac{d x_{2}}{x_{2}} d \tau \frac{T\left[Y^{n-4}\right]}{\left(Y_{1} . Y_{3}\right) \prod_{i \neq 1,2,3}\left(Y_{2} . Y_{i}\right)} \int \frac{\left\langle X d^{n-4} X\right\rangle 2 \rho d \rho}{\left(\rho^{2}+\sum_{i \neq 1,2,3} x_{i}\right)^{n-2}} .
$$

The integral $\int \frac{\left\langle X d^{n-4} X\right\rangle \rho d \rho}{\left(\rho^{2}+\sum_{i \neq 1,2,3} x_{i}\right)^{n-2}}=\frac{1}{2(n-3) !}$ is independent of the details of the numerator. This explains why $\Gamma_{I_{n}}$ is always rational at one-loop irrespective of the details of the integrand.

We have shown that the co-efficient of the IR divergence can be extracted from the integral by an algebraic operation directly in Feynman parameter space. There is a potential IR divergence associated with every triplet $\left(x_{i-1}, x_{i}, x_{i+1}\right)$. The complete IR divergence associated with the one-loop integral (3.10) is given by summing over all such regions

$$
\Gamma=\sum_{i=1}^{n} \frac{T\left[Y_{i}^{n-4}\right]}{2\left(Y_{i-1} \cdot Y_{i+1}\right) \prod_{k \neq i-1, i, i+1} Y_{i} \cdot Y_{k}} .
$$




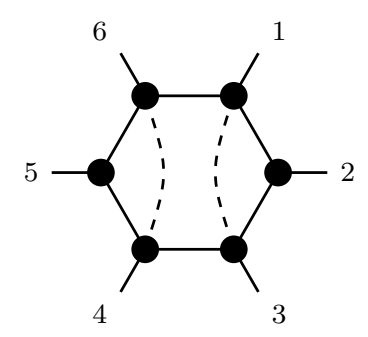

Figure 2. Chiral Hexagon.

\subsection{A basis for IR finite integrals in Feynman parameter space}

It is instructive to understand what makes integrals IR finite in Feynman parameter space. We can see from eq. (3.12) that $\Gamma=0$ unless $T\left[Y_{i}^{n-4}\right] \neq 0$ for at least one $i \in\{1, \ldots n\}$. As an example, consider a well known finite integral, the chiral hexagon (figure 2).

$$
I=\int_{A B} \frac{\langle A B 13\rangle\langle A B 46\rangle\langle 5612\rangle\langle 2345\rangle}{\langle A B 12\rangle\langle A B 23\rangle\langle A B 34\rangle\langle A B 45\rangle\langle A B 56\rangle\langle A B 16\rangle}
$$

where we have used momentum twistor notation and $\int_{A B}=\int\left\langle A B d^{2} A\right\rangle\left\langle A B d^{2} B\right\rangle$. On Feynman parametrization, this becomes, ${ }^{2}$

$$
\frac{\left(Y_{13} . Y_{46}\right)(W . W)-6\left(W . Y_{13}\right)\left(W . Y_{46}\right)}{(W . W)^{4}} .
$$

where $W=\sum_{i} x_{i} Y_{i}$ and $Y_{i j}$ is the vector in embedding space corresponding to the bitwistor $|i j\rangle$. The numerator doesn't contain any terms of the form $x_{i}^{2}$ and doesn't lead to IR divergences from the soft-collinear region.

We can now easily construct a basis of IR finite integrals in Feynman parameter space. At n-points, the numerator of a Feynman integral is a polynomial of degree $(n-4)$ in the Feynman parameters.

$$
\int\left\langle X d^{n-1} X\right\rangle \frac{T\left[X^{n-4}\right]}{(X Q X)^{(n-2)}}
$$

where $T\left[X^{n-4}\right]=T_{i_{1} \ldots i_{n-4}} X^{i_{1} \ldots i_{n-4}}$. The only constraint IR finiteness imposes on $\mathrm{T}$ is that coefficients of $x_{i}^{n-4}$ should vanish for all $i \in\{1, \ldots n\}$.

At $n=5$, this implies that there are no IR finite integrals. This is in agreement with the result that the chiral pentagons for $n=5$ suffer from IR divergences from unprotected massless corners [9].

At $n=6$, the tensor is left with 15 independent coefficients. Further conditions can be imposed to uniquely specify a basis. For instance, we can demand that some leading singularities vanish while others are \pm 1 . We will develop these ideas further in section 5 . But first, we need to understand the avatar of leading singularities in Feynman parameter space, which involve the notion of spherical contours.

\footnotetext{
${ }^{2}$ This Feynman parametriztion has been worked out in eq. (5.20) of [1].
} 


\section{Algebraic aspects of spherical residues}

The idea of a spherical contour integral and the corresponding spherical residue was introduced in [1] to compute the discontinuities of one-loop integrands directly in Feynman parameter space. Here, we briefly review the central result of section[6] of [1] before proceeding to analyze the transformation of numerators under a spherical residue. Consider the following integral.

$$
I_{n, k}=\int \frac{\left\langle X d^{n-1} X\right\rangle T\left[X^{k}\right]}{(X . Q \cdot X)^{\frac{(n+k)}{2}}}
$$

with $k$ and $n$ even. For any pair of Feynman parameters $\left(x_{i}, x_{j}\right)$, there is a natural decomposition of the quadric $Q$ into four parts,

$$
\begin{aligned}
X \cdot Q \cdot X= & X_{\{i j\}} \cdot Q_{\{i j\},\{i j\}} \cdot X_{\{i j\}}+X_{\{i j\}} \cdot Q_{\{i j\},\{\hat{i j}\}} \cdot X_{\{\hat{i j}\}}+ \\
& X_{\{\hat{i j}\}} \cdot Q_{\{\hat{i j}\},\{i j\}} X_{\{i j\}}+X_{\{\hat{i j}\}} \cdot Q_{\{\hat{i j}\},\{\hat{i j}\}} \cdot X_{\{\hat{i j}\}}
\end{aligned}
$$

where

$$
\begin{aligned}
& X_{\{i j\}}=\left(x_{i}, x_{j}\right) \\
& X_{\{\hat{i j}\}}=\left(x_{1}, \ldots \hat{x}_{i}, \ldots, \hat{x}_{j} \ldots x_{n}\right)
\end{aligned}
$$

and the $\hat{x}_{i}$ indicates that the entry is missing. The integral can develop singularities at locations determined by the entries of $Q$ (which are functions of the external momenta) and the properties of the numerator. There are possible branch point beginning at the following locations (see the text around eq. (6.23) of [1] for more explanation).

$$
\overline{i j}= \begin{cases}r\left(Q_{\{i j\},\{i j\}}^{-1}\right) & Q_{i i} \neq 0, Q_{j j} \neq 0 \\ \left(\frac{Q_{i j}^{2}}{Q_{j j}}\right)^{-\operatorname{sign}\left(Q_{i j}\right)} & Q_{i i}=0, Q_{j j} \neq 0 \\ \left(\frac{Q_{i j}^{2}}{Q_{i i}}\right)^{-\operatorname{sign}\left(Q_{i j}\right)} & Q_{i i} \neq 0, Q_{j j}=0 \\ Q_{i j}^{-2} \operatorname{sign}\left(Q_{i j}\right) & Q_{i i}=0, Q_{j j}=0\end{cases}
$$

where $r(\mathbf{A})=\frac{\mathbf{A}_{12}-\sqrt{\left(\mathbf{A}_{12}\right)^{2}-\mathbf{A}_{11} \mathbf{A}_{22}}}{\mathbf{A}_{12}+\sqrt{\left(\mathbf{A}_{12}\right)^{2}-\mathbf{A}_{11} \mathbf{A}_{22}}}$ and sign $\left(Q_{i j}\right)$ is just the sign of the element $Q_{i j}$. These are actual branch points only if the residue on the spherical contour corresponding to the variables $x_{i}$ and $x_{j}$ is non zero. In the cases when the residue is non-zero, its value gives the discontinuity across the cut.

To compute the spherical residue, we use the following algorithm.

- Perform the transformation

$$
\left(\begin{array}{c}
x_{i} \\
x_{j}
\end{array}\right)=R\left(\begin{array}{c}
w_{i} \\
w_{j}
\end{array}\right)-Q_{\{i j\}\{i j\}}^{-1} Q_{\{i j\}\{\hat{i j}\}} X_{\{\hat{i}\}} .
$$


thereby reducing the denominator to the form

$$
w_{i} w_{j}+X_{\{\widehat{i j}\}} Q^{(i j)} X_{\{\widehat{i j}\}}
$$

Here $R$ is a $2 \times 2$ matrix such that $R^{T} Q_{\{i j\}\{i j\}} R=\left(\begin{array}{cc}0 & 1 / 2 \\ 1 / 2 & 0\end{array}\right)$.

- Integrate over the entire complex plane / Riemann sphere by setting $w_{i}=r e^{i \theta}$ and $w_{j}=r e^{-i \theta}$ with ranges $r \in(0, \infty)$ and $\theta \in(0,2 \pi)$.

It was shown in [1] (see eq. (6.10)) that the whole procedure can be interpreted as an algebraic operation on the quadric, i.e. after integration the new quadric $Q^{(i j)}$ is related to the old one by

$$
Q^{(i j)}=Q_{\{\widehat{i j}\}\{\hat{i j}\}}-Q_{\{\hat{i j}\}\{i j\}} Q_{\{i j\}\{i j\}}^{-1} Q_{\{i j\}\{\hat{i j}\}} .
$$

Furthermore, the effect of performing multiple spherical contour integrals is captured by extensions of the same formula. In 4 spacetime dimensions, the maximum number of spherical contours we can perform is two (this is equivalent to cutting four propagators and fully localizing the momentum). This double spherical residue results in a quadric

$$
Q^{(i j k l)}=Q_{\{\widehat{i j k l}\}\{\widehat{i j k l}\}}-Q_{\{\widehat{i j k l}\}\{i j k l\}} Q_{\{i j k l\}\{i j k l\}}^{-1} Q_{\{i j k l\}\{\widehat{i j k l}\}} .
$$

In order to complete the interpretation as an algebraic operation, we need to provide similar expressions for the numerators of the integrals. We will now examine the effect the spherical contour integral has on the numerators.

Linear numerator. Let's start with a Feynman parameter integral with a linear numerator,

$$
I_{l}=\int \frac{\left\langle X d^{n-1} X\right\rangle(L \cdot X)}{(X \cdot Q \cdot X)^{(n+1) / 2}} .
$$

We want an expression for the numerator after performing a spherical contour integral along the $\left(x_{i}, x_{j}\right)$. To perform the integral, we first decompose the numerator into parts along $x_{i}, x_{j}$ and orthogonal pieces.

$$
L \cdot X=L_{\widehat{\{i j\}}} \cdot X_{\widehat{\{i j\}}}+L_{\{i j\}} \cdot\left(x_{i}, x_{j}\right) .
$$

Performing the transformation eq. (4.2) results in an integral which we denote as

$$
I_{l}^{(i j)}=\int \frac{\left\langle X_{\{\widehat{i j}\}} d^{n-3} X_{\{\widehat{i j}\}}\right\rangle\left(L^{(i j)} \cdot X_{\{\widehat{i j}\}}\right)}{\left(X_{\{\hat{i j}\}} \cdot Q^{(i j)} \cdot X_{\{\widehat{i j}\}}\right)^{(n-1) / 2}}
$$

with

$$
L^{(i j)}=\frac{1}{\sqrt{-4 \operatorname{Det} Q_{\{i j\}\{i j\}}}}\left(L_{\widehat{\{i j\}}}-L_{\{i j\}} Q_{\{i j\}\{i j\}}^{-1} Q_{\{i j\}\{\hat{i j}\}}\right)
$$


Quadratic numerator. Consider next, the case of an integral with a quadratic numerator.

$$
I_{q}=\int \frac{\left\langle X d^{n-1} X\right\rangle(N \cdot X \cdot X)}{(X \cdot Q \cdot X)^{(n+2) / 2}}
$$

To perform a spherical contour integral in the $\left(x_{i}, x_{j}\right)$ direction, we decompose $N^{(i j)}$ in the same way as before.

$$
X . N . X=X_{\{i j\}} N_{\{i j\}\{i j\}} X_{\{i j\}}+2 N_{\{i j\}\{\hat{i}\}} X_{\{i j\}} X_{\{\hat{i j}\}}+N_{\{\widehat{i j}\}\{\hat{i j}\}} X_{\{\hat{i j}\}} X_{\{\hat{i j}\}} .
$$

We can show that the result can be written as

$$
I_{q}^{(i j)}=\int \frac{\left\langle X^{(i j)} d^{n-3} X^{(i j)}\right\rangle\left(X^{(i j)} \cdot N^{(i j)} \cdot X^{(i j)}\right)}{\left(X^{(i j)} \cdot Q^{(i j)} \cdot X^{(i j)}\right)^{n / 2}}
$$

with

$$
\begin{aligned}
N^{(i j)}= & Q^{(i j)} \operatorname{Tr}\left(Q_{\{i j\}\{i j\}}^{-1} N_{\{i j\}\{i j\}}\right)+(n-2)\left(Q_{\{\hat{i j}\}\{i j\}} Q_{\{i j\}\{i j\}}^{-1} N_{\{i j\}\{i j\}} Q_{\{i j\}\{i j\}}^{-1} Q_{\{i j\}\{\hat{i j}\}}\right. \\
& \left.-Q_{\{\hat{i j}\}\{i j\}} Q_{\{i j\}\{i j\}}^{-1} N_{\{i j\}\{\hat{i j}\}}-N_{\{\widehat{i j}\}\{i j\}} Q_{\{i j\}\{i j\}}^{-1} Q_{\{i j\}\{\hat{i j}\}}+N_{\{\widehat{i j}\}\{\widehat{i j}\}}\right) .
\end{aligned}
$$

For more details on the calculation, we refer the reader to appendix B.

The effect of multiple spherical contours is easy to express in this form. For e.g. a double spherical residue along directions $(i j k l)$, on the linear and quadratic numerators, results in $L^{(i j k l)}$ and $N^{(i j k l)}$ with obvious definitions.

\subsection{Properties of Feynman integrals coming from loop integrals}

In this section we elaborate on some properties satisfied by Feynman integrals. An integral of the form given in eq. (4.1) must satisfy the following conditions if it comes from a Feynman diagram.

- The quadric $Q$ must be degenerate for $n>6$. This is because the entries of the quadric are all of the form $Y_{i} . Y_{j}$ where $Y_{i}$ and $Y_{j}$ are embedding space vectors. The embedding space corresponding to $4 \mathrm{D}$ spacetime is 6 dimensional. Thus the rank of $\mathrm{Q}$ is always 6 .

- The tensor in the numerator, $T$ must share the null space of the degenerate $\mathrm{Q}$ (for $n>6$ ). If $N$ is a vector in the null space of $Q$, i.e. $Q \cdot N=0$, then we must have $T . N=0$.

It is a non trivial fact that these properties continue to hold after we perform a spherical contour integral. We can use the expressions derived above to provide a quick proof of these facts.

This is easy to show for a Feynman parameter integral with a linear numerator eq. (4.3). We want to show that the new numerator shares a null space with the new quadric, i.e. for every $N^{\prime}$ such that $Q^{(i j)} . N^{\prime}=0$, we have $L^{\prime} . N=0$. To show this, suppose that $N$ belongs 
to the null space of $L$ and $Q$. Then we have $L \cdot N=0=Q \cdot N=0$. It is easy to see that $N^{\prime}=N_{\{\hat{i j}\}}$ is a null vector of $Q^{(i j)}$ using the following property.

$$
Q . N=0 \Longrightarrow Q_{\{\}\{i j\}} N_{\{i j\}}=-Q_{\{\}\{\widehat{i j}\}} N_{\{\hat{i} j\}}
$$

where the empty \{\} can be either $\{i j\}$ or $\{\widehat{i j}\}$. Using eq. (4.5) it is obvious that $L^{(i j)} \cdot N^{\prime}=0$. Thus eq. (4.3) satisfies all the conditions of a Feynman integral after a spherical contour.

This can be extended to a class of integrals of the form

$$
\left\langle X d^{n-1} X\right\rangle \frac{(L \cdot X)^{n-D}}{(X \cdot Q \cdot X)^{n-D / 2}}
$$

The spherical residue in variables $\left(x_{i}, x_{j}\right)$ is a sum of terms of the form $(0 \leq k \leq n-D)$

$$
\frac{\left(L^{(i j)} \cdot X\right)^{n-D-k / 2}}{\left(X^{\prime} Q^{\prime} X^{\prime}\right)^{(D+k+2-2 n) / 2}} .
$$

See A for the detailed derivation of this result. We see that the proof for a linear numerator works here as well. An similar calculation using eq. (4.7) shows that the same holds true in the case of a quadratic numerator

\subsection{Spherical contours meet IR divergences}

We have seen that the double spherical contours calculate the leading singularities. We know that leading singularities obey relations that arise from the global residue theorem [9]. These must be reflected in the double spherical contours. Let us start with the simple example of

$$
I_{5}=\int \frac{\left\langle X d^{4} X\right\rangle x_{2}}{\left(x_{1} x_{3} Q_{13}+x_{1} x_{4} Q_{14}+x_{2} x_{4} Q_{24}+x_{2} x_{5} Q_{25}+x_{3} x_{5} Q_{35}\right)^{3}} .
$$

This integral is IR divergent and the divergence corresponds to the triplet $\left(x_{1} x_{2} x_{3}\right)$. Let us calculate the double spherical contours (1423), (1425), (1324), (1325).

$$
c_{1423}=-c_{1425}=c_{1324}=c_{1325}=-\frac{1}{2 Q_{25} Q_{13} Q_{24}} .
$$

We see that $c_{1425}+c_{1423}=0$ as expected from the Global residue theorem. However, $c_{1324}+c_{1325} \neq 0$ and this is precisely because of the IR divergence. Similar residue theorems are satisfied by the double spherical contours as can be checked from our expression for the 6 point MHV amplitude. Since the IR divergence introduces non-zero composite residues, the statement of the global residue theorem must be changed to accommodate these. The spherical residue capture the usual leading singularities in Feynman parameter space and the scaling limit introduced in section 3.2 captures the composite residues. A similar analysis can be found in [30]. 


\section{Constructing integrands using spherical residues}

In $4 D$, performing two spherical contour integrals is equivalent to putting four propagators on-shell. This fully localizes the loop momentum. The resulting object is the sum of the leading singularities associated with cutting the four propagators. Specifying the leading singularities (LS) puts constraints on the integrand. We can construct integrands from their singularities in Feynman parameter space using this technique. In this section, we will illustrate this with a few examples at 5 and 6 points. We will use our knowledge of the leading singularities of MHV amplitudes of $\mathcal{N}=4$ SYM to construct the one-loop integrand for the 5 and 6 point amplitudes.

\subsection{5 point integrands}

At 5 points, a generic Feynman parameter integrand is

$$
I_{5}=\int\left\langle X d^{4} X\right\rangle \frac{(L \cdot X)}{(X \cdot Q \cdot X)^{3}} .
$$

Since we know that the only allowed poles in momentum twistor space are of the form $\langle A B i i+1\rangle=0$, we will assume that the quadric is $Q_{i j}=\langle i-1 i j-1 j\rangle$. The vector in the numerator $L=\left(l_{1}, l_{2}, l_{3}, l_{4}, l_{5}\right)$ is to be determined from the LS. We demand that all the LS are equal and for convenience, we set them equal to 1.

We have five unique double spherical contour integrals corresponding to the five one mass LS. We denote a double spherical residue by the four associated Feynman parameters. (Note that our Feynman parameters are labeled such that the contour $(i j)$ is equivalent to cutting propagators $\langle A B i-1 i\rangle=\langle A B j-1 j\rangle=0$.) The residue corresponding to (1435) is

$$
\frac{2\left(l_{5} Q_{13} Q_{24}+l_{4} Q_{13} Q_{25}-l_{3} Q_{14} Q_{25}+l_{2} Q_{14} Q_{35}-l_{1} Q_{24} Q_{35}\right)}{Q_{13} Q_{24} Q_{25}} .
$$

Demanding that this be 1 imposes a constraint on the $l_{i}$. Similarly demanding that all the other LS are equal to one leads to the numerator

$$
l=1 / 2\left(Q_{13} Q_{14} Q_{25}, Q_{13} Q_{24} Q_{25}, Q_{13} Q_{24} Q_{35}, Q_{14} Q_{24} Q_{35}, Q_{14} Q_{25} Q_{35}\right) .
$$

We see that the leading singularities completely determine the five point amplitude in Feynman parameter space. This should be compared with eq. (6.7) which was obtained by summing all the chiral pentagons at 5 points. Note that this integrand is IR divergent and has all the divergences associated with the 5 point amplitude.

We can also construct an integrand with only one non zero LS. Demanding that $I_{5}$ has support only on the cut (1425) and has unit residue results in

$$
\int\left\langle X d^{4} X\right\rangle \frac{Q_{14} Q_{25}}{4} \frac{\left(x_{1} Q_{13}+x_{5} Q_{35}\right)}{(X Q X)^{3}} .
$$

It is easy to recognize that this is the Feynman parametrization of

$$
\int_{A B} \frac{\langle A B 34\rangle}{\langle A B 12\rangle\langle A B 23\rangle\langle A B 34\rangle\langle A B 45\rangle\langle A B 15\rangle} .
$$




\subsection{6 point integrands}

A generic 6 point integrand in Feynman parameter space has a quadratic numerator.

$$
I_{6}=\int\left\langle X d^{5} X\right\rangle \frac{X \cdot N \cdot X}{(X \cdot Q \cdot X)^{4}} .
$$

$N$ is a symmetric, rank 2 tensor. The quadric $Q_{i j}=\langle i-1 i j-1 j\rangle$ as usual for a one-loop integral. We can always make a change of variables to reduce it to

$$
Q=\left(\begin{array}{cccccc}
0 & 0 & 1 & 1 & 1 & 0 \\
0 & 0 & 0 & u_{1} & 1 & 1 \\
1 & 0 & 0 & 0 & u_{2} & 1 \\
1 & u_{1} & 0 & 0 & 0 & u_{3} \\
1 & 1 & u_{2} & 0 & 0 & 0 \\
0 & 1 & 1 & u_{3} & 0 & 0
\end{array}\right)
$$

We refer the reader to appendix $\mathrm{C}$ for more details. We have three kinds of leading singularities, one-mass, two-mass easy and two-mass hard. All the two mass hard leading singularities must vanish and all the remaining ones must be equal. We normalize them to unity for convenience. For computational simplicity, we choose external data

$$
Z_{n}=\left(1, n, n^{2}, n^{3}\right), \quad n=1, \ldots 6 .
$$

The constraints on $N$ arising from specifying the leading singularities suffice to fix all but 6 of the coefficients. After implementing these constraints, the integral can be written as a sum of two terms.

$$
I_{6}=\int\left\langle X d^{5} X\right\rangle \frac{\left(X . N_{1} \cdot X+X . N_{2} \cdot X\right)}{(X . Q . X)^{4}}
$$

with

$$
\begin{aligned}
X . N_{1} . X= & 9\left(729 x_{1}^{2}+810 x_{1} x_{2}+81 x_{2}^{2}+126 x_{2} x_{3}+45 x_{3}^{2}+50 x_{3} x_{4}+5 x_{4}^{2}-648 x_{2} x_{5}\right. \\
& \left.+495 x_{3} x_{5}+50 x_{4} x_{5}+45 x_{5}^{2}+810 x_{1} x_{6}+1215 x_{3} x_{6}+207 x_{4} x_{6}+126 x_{5} x_{6}+81 x_{6}^{2}\right) \\
X . N_{2} . X= & 2 n_{13} x_{1} x_{3}+2 n_{14} x_{1} x_{4}+2 n_{24} x_{2} x_{4}+2 n_{15} x_{1} x_{5}-2 n_{14} x_{2} x_{5}+2 n_{15} x_{2} x_{5}+18 n_{24} x_{2} x_{5} \\
& -\left(10 n_{14} x_{3} x_{5}\right) / 9-\left(10 n_{26} x_{3} x_{5}\right) / 9+2 n_{26} x_{2} x_{6}-2 n_{15} x_{3} x_{6}-18 n_{24} x_{3} x_{6} \\
& -\left(2 n_{13} x_{4} x_{6}\right) / 9+\left(2 n_{14} x_{4} x_{6}\right) / 9-\left(2 n_{15} x_{4} x_{6}\right) / 9-2 n_{24} x_{4} x_{6} .
\end{aligned}
$$

The large integers that arise in this expression are due to the choice of external data. It is tedious but possible to rewrite this expression in terms of $\langle i j k l\rangle$. The integral with numerator $X . N_{2} . X$ is always rational and all its double spherical residues vanish. Here, we see a clear separation in Feynman parameter space of the rational part and the transcendental part. 


\section{Feynman parametrization in planar $\mathcal{N}=4 \mathrm{SYM}$}

In this section, we examine the one-loop MHV integrand of $\mathcal{N}=4$ SYM. It is completely determined by its leading singularities and has a well known expression in terms of chiral pentagons.

$$
\mathcal{A}_{\mathrm{MHV}}^{1 \text {-loop }}=\sum_{i<j<i}\{\underbrace{i}_{\star r^{\circ}}\} \text {. }
$$

Henceforth, we denote the chiral pentagon integral shown above by $(j i)$ which takes the following form in momentum twistor space.

$$
(j i)=\int_{A B} \frac{\langle A B(j-1 j j+1) \cap(i-1 i i+1)\rangle\langle\star j i\rangle}{\langle A B i-1 i\rangle\langle A B i i+1\rangle\langle A B j-1 j\rangle\langle A B j j+1\rangle\langle A B \star\rangle}
$$

where $\star$ is an arbitrary bi-twistor.

There are two leading singularities, i.e. two solutions to the set of equations

$$
\langle A B i-1 i\rangle=\langle A B i i+1\rangle=\langle A B j-1 j\rangle=\langle A B j j+1\rangle=0 .
$$

These are the lines $Z_{[i} Z_{j]}$ and $(i-1 i i+1) \cap(j-1 j j+1)$. The above integrand is chiral and has vanishing support on the solution $(i-1 i i+1) \cap(j-1 j j+1)$. Thus an individual chiral pentagon is tailored to reproduce a leading singularity. However, it also has additional leading singularities arising from the pole $\langle A B \star\rangle$. These are not singularities of the amplitude and must cancel in the sum in eq. (6.1). The cancellation of the spurious poles is not manifest and it is desirable to obtain an expression for the complete amplitude which is free of spurious poles. For attempts along this line in momentum twistor space, see [31]. Here, we will derive an expression for the complete integrand in Feynman parameter space and we will see a transparent cancellation of the spurious poles. We begin with the simple case of the four point amplitude. In this case, there are 12 contributing pentagons

$$
\begin{aligned}
\mathcal{A}_{4}^{\mathrm{MHV}} & =(1,2)+(1,3)+(1,4)+\text { cyclic } \\
\mathcal{A}_{4}^{\mathrm{MHV}} & =\int_{A B} \frac{2\langle 1234\rangle}{\langle A B 12\rangle\langle A B 23\rangle\langle A B 34\rangle\langle A B 41\rangle\langle A B \star\rangle} \times\left\{\begin{array}{l}
-\langle\star 12\rangle\langle A B 34\rangle+\langle\star 23\rangle\langle A B 41\rangle \\
-\langle\star 34\rangle\langle A B 12\rangle+\langle\star 41\rangle\langle A B 23\rangle \\
+\langle A B 24\rangle\langle\star 13\rangle+\langle A B 13\rangle\langle\star 24\rangle
\end{array}\right\} \\
& =\int\left[d^{4} Y\right]\left\langle X d^{4} X\right\rangle \frac{(Y . N)}{(W . Y)^{5}}
\end{aligned}
$$

where

$$
W=x_{1}|12\rangle+x_{2}|23\rangle+x_{3}|34\rangle+x_{4}|41\rangle+x|\star\rangle
$$

and $N$ is the numerator of eq. (6.3) written in embedding space. Performing the momentum integral yields the Feynman parametrization.

$$
\mathcal{A}_{4}^{\mathrm{MHV}}=\int\left\langle X d^{4} X\right\rangle \frac{1}{(W . W)^{3}}\left\{\begin{array}{c}
-2\langle 1234\rangle x(\langle\star 13\rangle\langle\star 24\rangle-\langle X 12\rangle\langle\star 34\rangle+\langle\star 23\rangle\langle\star 41\rangle) \\
+\langle 1234\rangle^{2}\left(x_{1}\langle\star 12\rangle+x_{2}\langle\star 23\rangle+x_{3}\langle\star 34\rangle+x_{4}\langle\star 41\rangle\right)
\end{array}\right\} .
$$


Having obtained the Feynman parametrization, it is now straightforward to demonstrate that $\mathcal{A}_{4}^{\mathrm{MHV}}$ is independent of both $x$ and $\star$. First, note that the coefficient of $x$, which is quadratic in $\star$ vanishes due to a Schouten identity. The rest of the expression can be written as a total derivative.

$$
\mathcal{A}_{4}^{\mathrm{MHV}}=-\frac{1}{2} \int\left\langle X d^{4} X\right\rangle\langle 1234\rangle^{2} \frac{\partial}{\partial x}\left(\frac{1}{(W . W)^{2}}\right)=\frac{1}{2} \int\left\langle X d^{3} X\right\rangle \frac{1}{(\tilde{W} \cdot \tilde{W})^{2}}
$$

with $\tilde{W}=\left.W\right|_{x=0}$ and the integral over the remaining Feynman parameters.

This procedure can be repeated at higher points. In each case, we find that the coefficient of the highest power of $x$ vanishes due to a Schouten identity and the rest can be written as a total derivative which is independent of $\star$ at the boundaries. We present an expression for the 5 point amplitude. The details of the calculations are relegated to appendix D.

$$
\mathcal{A}_{5}^{\mathrm{MHV}}=\int\left\langle X d^{5} X\right\rangle \frac{\partial}{\partial x}\left(\frac{2 n_{0}(W \cdot \star)+\tilde{W} \cdot \tilde{W} n_{1}+3(W \cdot \star) n_{1} x}{(\tilde{W} \cdot \tilde{W})^{3}(W \cdot \star)^{2}}\right) .
$$

Here $n_{0}$ and $n_{1}$ are the coefficients of $x^{0}$ and $x$ in eq. (D.2). As before, the integral localizes to the boundaries where it is independent of the bi-twistor $\star$ and is given by

$$
\begin{aligned}
\mathcal{A}_{5}^{\mathrm{MHV}}= & \int\left\langle X d^{4} X\right\rangle \frac{n}{d^{3}} \\
n= & \left(\langle 1234\rangle\langle 1245\rangle\langle 1235\rangle x_{1}+\langle 1234\rangle\langle 2345\rangle\langle 1235\rangle x_{2}+\langle 1345\rangle\langle 1234\rangle\langle 2345\rangle x_{3}\right. \\
& \left.+\langle 1345\rangle\langle 2345\rangle\langle 1245\rangle x_{4}+\langle 1345\rangle\langle 1245\rangle\langle 1235\rangle x_{5}\right) \\
d= & \tilde{W} \cdot \tilde{W} \quad \text { where } \quad \tilde{W}=x_{1}|12\rangle+x_{2}|23\rangle+x_{3}|34\rangle+x_{4}|45\rangle+x_{5}|15\rangle .
\end{aligned}
$$

It is easy to see that eq. (6.4) and eq. (6.7) have the correct singularity structure. The presence of linear terms in the numerator of the 5 point amplitude implies the presence of IR divergences as expected. We can obtain similar expressions for the integrand at higher points. However, this has to be done on a case by case basis and we don't have a general expression.

\section{Outlook}

In this paper we have explored the singularity structure of one-loop Feynman parameter integrands and their geometry. The spherical residue captures the notion of discontinuity and the double spherical residue that of leading singularities. Feynman parameter integrands that arise from Feynman graphs satisfy special constraints and we saw that the spherical contour remarkably preserves these properties. We have provided an algebraic description of spherical residues and given formulae which can be use to compute both them as algebraic mappings. The double spherical residue was exploited to construct Feynman parameter integrands. Composite residues in momentum space captures the leading IR divergences. The scaling procedure introduced in section 3.2 to extract the leading IR divergences shows that the notion of composite residues exists even in Feynman parameter space. 
The obvious next step is to extend the results of this paper beyond one loop. It would be interesting to explore the extraction of the leading IR divergence of a two loop graph by a similar method. For some details on higher loop Feynman parametrization and IR divergences, we draw the reader's attention to [32]. While extraction of the leading IR behaviour is fascinating in its own right, it could also prove useful in calculating the cusp anomalous dimension of $\mathcal{N}=4 \mathrm{SYM}$ which has been a topic of some interest in the past few years [18-21]. The knowledge of the relationship between cuts of Feynman graphs and discontinuities is intensely studied in momentum space (see [33, 34]). In Feynman parameter space, this amounts to an understanding of the relationship between spherical residues and leading singularities at higher loops. This is an essential ingredient in attempting any construction of higher loop integrands. While these are some of the immediate pragmatic questions of general interest, some features of Feynman parameter integrands of $\mathcal{N}=4$ SYM raise more provocative questions.

Section 6 shows the explicit independence of MHV amplitudes on spurious poles at 4 and 5 points. While this cancellation is expected even in momentum twistor space, it is simpler to observe in Feynman parameter space and isn't the consequence of a complicated identity satisfied by the external data. Another miraculous feature, seen from the 4 and 5 point one-loop integrands, eq. (6.4) and eq. (6.7), is that they are both manifestly positive (for positive external data). Positivity of the integrands in momentum twistor space was observed in [31]. There the positivity stemmed from the more complicated identity $\langle A B \bar{i} \bar{j}\rangle>0$ for configurations of $Z_{i}$ in the amplituhedron. Here, $\langle i j k l\rangle>0$ for $i<j<k<l$ suffices to guarantee positivity. It is crucial to check if these features persist beyond one loop. It would also be interesting to analyze the positivity properties of the log of the amplitude and the n-point Ratio function [35] in Feynman parameter space.

The existence of these properties seems to suggest that Feynman parameter space more than an auxiliary space introduces to aid in integration and is a natural space to study loop integrands. In the last decade, a rich geometric structure underlying scattering amplitudes of $\mathcal{N}=4$ SYM has been uncovered [36, 37] and positive geometry [38] is at the heart of it all. It is a natural to wonder if the properties seen here are a reflection of this structure. If this were true, it suggests that Feynman parameter space has an extremely rich geometry and the properties observed thus far are only the tip of the iceberg.

\section{Acknowledgments}

We would like to thank Nima Arkani-Hamed for guidance at all stages of this project and for going through multiple versions of the manuscript. We also thank Ellis Yuan and Enrico Herrmann for useful discussions.

\section{A Cuts of Feynman integrals}

A class of integral coming from Feynman parametrizing a 1-loop diagram will are of the form

$$
I_{n}=\int\left\langle X d^{n-1} X\right\rangle \frac{(L \cdot X)^{n-D}}{(X \cdot Q \cdot X)^{n-D / 2}} .
$$


We will perform a spherical contour integral in the $(i j)$ directions. Using the transformation in eq. (4.2), the above integral becomes,

$$
I_{n}^{(i j)}=\int d w_{i} d w_{j}\left\langle X^{(i j)} d^{n-3} X^{(i j)}\right\rangle \mathcal{R} \frac{\left(L_{\{\hat{i} j\}} X_{\{\hat{i} \hat{j}\}}+L_{\{i j\}}(R w)_{\{i j\}}-L_{\{i j\}} Q_{\{i j\}\{i j\}}^{-1} Q_{\{i j\}\{\hat{i}\}} X_{\{\hat{i}\}\}}\right)^{n-D}}{\left(w_{i} w_{j}+X^{(i j)} Q^{(i j)} X^{(i j)}\right)^{n-D / 2}}
$$

where $\mathcal{R}=\operatorname{det} R$. The integral over $w_{i}, w_{j}$ to be done over $S^{2}$ with an implicit factor of $\frac{1}{2 \pi i}$. Using eq. (4.5), we can write the numerator as

$$
\left(L_{\{i j\}} R w_{i j}+L^{(i j)} X_{\{\widehat{i j}\}}\right)^{n-D}
$$

Since we are integrating over the Riemann sphere with the substitution $w_{i}=r e^{i \phi}, w_{i}=$ $r e^{-i \phi}$, only terms containing some power of the product $w_{i} w_{j}$ survive the angular integration. This yields,

$$
\begin{aligned}
I_{n}^{(i j)}= & \sum_{k=0, \text { even }}^{n-D}\left(\begin{array}{c}
n-D \\
k
\end{array}\right)\left(\begin{array}{c}
k \\
k / 2
\end{array}\right)(R \cdot L)_{i}^{k / 2}(R \cdot L)_{j}^{k / 2} \frac{\Gamma(1+k / 2) \Gamma(-1-D / 2-k / 2+n)}{2 \Gamma(n-D / 2)} \\
& \int\left\langle X^{(i j)} d^{n-3} X^{(i j)}\right\rangle \frac{\left(L^{(i j)} \cdot X^{(i j)}\right)^{n-D-k / 2}}{\left(X^{(i j)} Q^{(i j)} X^{(i j)}\right)^{(D+k+2-2 n) / 2}} .
\end{aligned}
$$

\section{B Spherical contour with a quadratic numerator}

In this appendix, we sketch out the details of transformation of a quadratic numerator under a spherical residue. Consider the integral in eq. (4.6). The transformation eq. (4.2) changes the numerator to

$$
\begin{aligned}
X . N . X \rightarrow & \operatorname{det} R\left((R w) N_{\{i j\}\{i j\}}(R w)+X_{\{\hat{i j}\}} Q_{\{\hat{i j}\}\{i j\}} Q_{\{i j\}\{i j\}}^{-1} N_{\{i j\}\{i j\}} Q_{\{i j\}\{i j\}}^{-1} Q_{\{i j\}\{\hat{i j}\}} X_{\{\hat{i j}\}}\right. \\
& \left.-2 X_{\{\widehat{i j}\}} Q_{\{\hat{i}\}\}\{i j\}} Q_{\{i j\}\{i j\}}^{-1} N_{\{i j\}\{\hat{i j}\}} X_{\{\hat{i j}\}}+X_{\{\hat{i j}\}} N_{\{\hat{i}\}\{\hat{i}\}} X_{\{\widehat{i j j}\}}\right) .
\end{aligned}
$$

With $\operatorname{det} R=2 \sqrt{-\operatorname{det} Q_{\{i j\}\{i j\}}}$, we can write the cut integral as

$$
I_{q}^{(i j)}=\int \frac{\left\langle X^{(i j)} d^{n-3} X^{(i j)}\right\rangle}{2 \sqrt{-\operatorname{det} Q_{\{i j\}\{i j\}}}} \frac{d w_{i} d w_{j}}{2 \pi i} \frac{(R w) N_{\{i j\}\{i j\}}(R w)+X_{\{\hat{i j}\}} N^{\prime} X_{\{\hat{i j}\}}}{\left(w_{i} w_{j}+X^{(i j)} Q^{(i j)} X^{(i j)}\right)^{\frac{n}{2}+1}} .
$$

The first term integrates to

$$
\frac{1}{2 \pi i} \int_{w_{i}=\bar{w}_{j}} \frac{\left(w \cdot\left(R^{T} N R\right) \cdot w\right)\left\langle X^{(i j)} d^{n-3} X^{(i j)}\right\rangle d w_{i} d w_{j}}{\left(w_{i} w_{j}+X_{\{\hat{i j}\}} Q^{(i j)} X_{\{\hat{i j}\}}\right)^{\frac{n+2}{2}}}=\frac{\left\langle X^{(i j)} d^{n-3} X^{(i j)}\right\rangle \operatorname{Tr}\left(Q_{\{i j\}\{i j\}}^{-1} N_{\{i j\}\{i j\}}\right)}{2 \sqrt{-\operatorname{det} Q_{\{i j\}\{i j\}} n(n-2)\left(X_{\{\hat{i j j}\}} Q^{(i j)} X_{\{\hat{i j}\}}\right)^{\frac{n}{2}-1}}}
$$

and the second one to

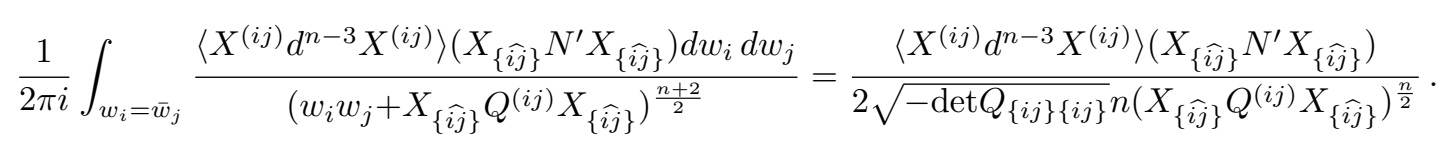



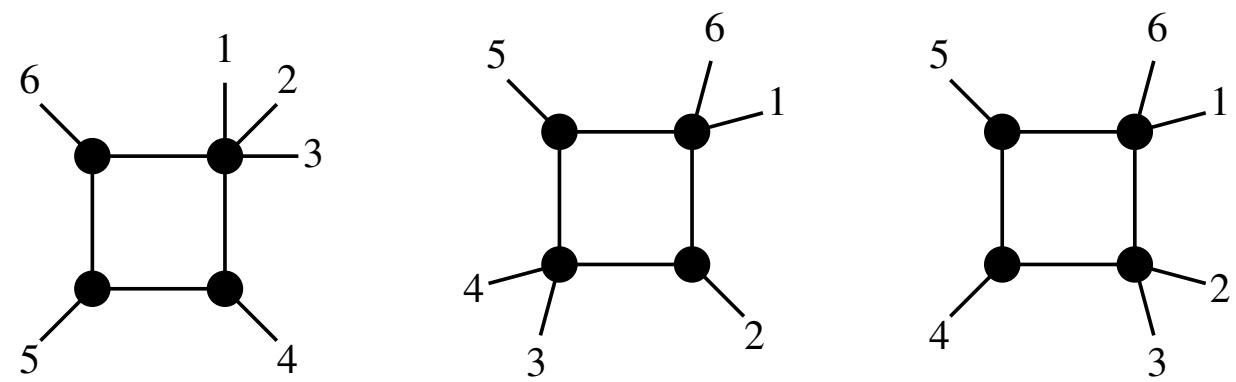

Figure 3. One-mass, two-mass easy and two-mass hard singularities.

\section{Leading singularities at 6 points}

At $n=6$, we can have leading singularities which correspond to the three box diagrams shown in figure 3 .

We label the leading singularities by the Feynman parameters of the cut propagators by associating $x_{i}$ with the propagator $\langle A B i i+1\rangle$. Thus $(i j k l)$ corresponds to the leading singularity which results from setting $\langle A B i-1 i\rangle=\langle A B j-1 j\rangle=\langle A B k-1 k\rangle=\langle A B l-1 l\rangle=$ 0 . In this notation, the list of singularities is

- One mass (1456), (3456), (1234), (6123), (5612), (2345)

- Two mass easy (1356), (6245), (5134), (4623), (3512), (2461)

- Two mass hard (3461), (4512), (5623)

The 1-loop, $n$-point amplitude of $\mathcal{N}=4 \mathrm{SYM}$ is a sum over all one-mass and two-mass easy leading singularities. Thus the numerator of the full amplitude is constrained to make all the two mass hard singularities vanish and to make all the other singularities equal. The six point amplitude in momentum twistor space must have the form

$$
\int_{A B} \frac{\langle A B X\rangle\langle A B Y\rangle}{\langle A B 12\rangle\langle A B 23\rangle\langle A B 34\rangle\langle A B 45\rangle\langle A B 56\rangle\langle A B 16\rangle}
$$

for some bi-twistors $\mathrm{X}$ and $\mathrm{Y}$. The corresponding object in Feynman parameter space looks like

$$
\int \frac{\left\langle X d^{5} X\right\rangle(X . N . X)}{(X . Q . X)^{4}}
$$

where the quadric $Q_{i j} \equiv q_{i j}=\langle i-1 i j-1 j\rangle$ and the numerator is a symmetric tensor with coefficients to be determined. We can simplify the denominator by making the transformation $x_{i} \rightarrow y_{i} x_{i}$ with

$$
y=\left(\sqrt{\frac{q_{25} q_{36}}{q_{13} q_{15} q_{26}}}, \sqrt{\frac{q_{15} q_{36}}{q_{13} q_{25} q_{26}}}, \sqrt{\frac{q_{15} q_{26}}{q_{13} q_{25} q_{36}}}, \sqrt{\frac{q_{13} q_{15} q_{26}}{q_{14}^{2} q_{25} q_{36}}}, \sqrt{\frac{q_{13} q_{26}}{q_{15} q_{25} q_{36}}}, \sqrt{\frac{q_{25} q_{13}}{q_{15} q_{26} q_{36}}}\right) .
$$

This transforms the denominator into X.Q.X $\rightarrow x_{1} x_{3}+x_{1} x_{4}+x_{1} x_{5}+u_{1} x_{2} x_{4}+x_{2} x_{5}+$ $x_{2} x_{6}+u_{2} x_{3} x_{5}+x_{3} x_{6}+u_{3} x_{4} x_{6}$ with $u_{1}=q_{24} q_{15} /\left(q_{25} q_{14}\right), u_{2}=q_{35} q_{26} /\left(q_{36} q_{25}\right)$ and $u_{3}=$ $q_{46} q_{13} /\left(q_{14} q_{36}\right)$.

We demand that all two-mass hard leading singularities vanish and that all the rest are equal to 1 . This places some constraints on the numerator $\mathrm{N}$. 


\section{Feynman parametrizing the MHV planar 1-loop integrand}

In this appendix, we provide the details of Feynman parametrizing the complete one-loop MHV integrand for planar $\mathcal{N}=4 \mathrm{SYM}$. As explained in section 6 , the one-loop integrand is given by

$$
\mathcal{A}_{n}^{\mathrm{MHV}}=\sum_{i<j<i}(j i)
$$

More concretely, the expression for the amplitude at $n$ points is

$$
\begin{aligned}
& \mathcal{A}_{n}^{\mathrm{MHV}}=\int_{A B}\left(\sum_{i=2}^{n} \frac{1}{\langle A B n 1\rangle\langle A B 12\rangle\langle A B \star\rangle} \frac{\langle A B(n 12 \cap i-1 i i+1)\rangle\langle\star 1 i\rangle}{\langle A B i-1 i\rangle\langle A B i i+1\rangle}\right)+\text { cyclic } \\
& \int_{A B}\left(\langle n 123\rangle\langle\star 12\rangle \prod_{i \neq n, 1,2}\langle A B i i+1\rangle-\langle 12 n-1 n\rangle\langle\star 1 n\rangle \prod_{i \neq 1, n-1, n}\langle A B i i+1\rangle\right. \\
& \mathcal{A}_{n}^{\mathrm{MHV}}=\frac{\left.+\sum_{k=2}^{n-1}\langle A B(n 12 \cap k-1 k k+1)\rangle\langle\star 1 k\rangle \prod_{i \neq k-1 k, 1, n}\langle A B i i+1\rangle\right)}{\langle A B \star\rangle \prod_{i=1}^{n}\langle A B i i+1\rangle} .
\end{aligned}
$$

Combining all the terms in the cyclic sum,

$$
\begin{aligned}
& \mathcal{A}_{n}^{\mathrm{MHV}}=\frac{1}{\langle A B \star\rangle \prod_{i=1}^{n}\langle A B i i+1\rangle}\left(2 \sum_{l=1}^{n}\langle\star l l+1\rangle \prod_{i \neq l-1, l, l+1}\langle A B i i+1\rangle\langle l-1 l l+1 l+2\rangle\right. \\
& \left.+\sum_{k=1}^{n} \sum_{l=k+2}^{k-2}\langle\star l k\rangle\langle A B(l-1 l l+1) \cap(k-1 k k+1)\rangle \prod_{i \neq k-1, k, l-1, l}\langle A B i i+1\rangle\right) .
\end{aligned}
$$

We can proceed with Feynman parametrization using embedding space techniques. The following formula is useful

$$
\frac{\left\langle A B Y_{1}\right\rangle \ldots\left\langle A B Y_{n-3}\right\rangle}{\langle A B 12\rangle \ldots\langle A B n 1\rangle\langle A B \star\rangle} \stackrel{F P}{\longrightarrow}\left(Y_{1} \cdot \frac{d}{d W}\right) \ldots\left(Y_{n-3} \cdot \frac{d}{d W}\right) \frac{1}{(W . W)^{2}}
$$

with $W=\sum_{i=1}^{n-1} x_{i}|i i+1\rangle+x_{n}|1 n\rangle+x|\star\rangle$.

In the 5 point case numerator after performing the cyclic sum is,

$$
\begin{aligned}
\langle\star 12\rangle & \langle 5123\rangle\langle A B 34\rangle\langle A B 45\rangle+\langle\star 23\rangle\langle 1234\rangle\langle A B 45\rangle\langle A B 51\rangle+\langle\star 34\rangle\langle 2345\rangle\langle A B 51\rangle\langle A B 12\rangle \\
& +\langle\star 45\rangle\langle 3451\rangle\langle A B 12\rangle\langle A B 23\rangle+\langle\star 51\rangle\langle 4512\rangle\langle A B 23\rangle\langle A B 34\rangle+\langle\star 13\rangle\langle A B \overline{1} \overline{3}\rangle\langle A B 45\rangle \\
& +\langle\star 14\rangle\langle A B \overline{1} \overline{4}\rangle\langle A B 23\rangle+\langle\star 24\rangle\langle A B \overline{2} \overline{4}\rangle\langle A B 51\rangle+\langle\star 25\rangle\langle A B \overline{2} \overline{5}\rangle\langle A B 34\rangle \\
& +\langle\star 35\rangle\langle A B \overline{3} \overline{5}\rangle\langle A B 12\rangle .
\end{aligned}
$$

Eq. (D.1) adapted to this case reads,

$$
\frac{\left(Y_{1} . Y_{2}\right)(W . W)-6\left(Y_{1} . W\right)\left(Y_{2} \cdot W\right)}{(W . W)^{3}}
$$


which yields the following Feynman parametrization for $\mathcal{A}_{5}^{\mathrm{MHV}}$.

$$
\begin{aligned}
\langle\star 12 & \langle\langle 5123\rangle(-6\langle W 34\rangle\langle W 45\rangle)+\langle\star 23\rangle\langle 1234\rangle(-6\langle W 45\rangle\langle W 51\rangle) \\
& +\langle\star 34\rangle\langle 2345\rangle(-6\langle W 51\rangle\langle W 12\rangle)+\langle\star 45\rangle\langle 3451\rangle(-6\langle W 12\rangle\langle W 23\rangle) \\
& +\langle\star 51\rangle\langle 4512\rangle(-6\langle W 23\rangle\langle W 34\rangle) \\
& +\langle\star 13\rangle[\langle 5124\rangle\langle 2345\rangle W . W-6\langle W 45\rangle(\langle W 12\rangle\langle 5234\rangle+\langle W 25\rangle\langle 1234\rangle)] \\
& +\langle\star 14\rangle[-\langle 5123\rangle\langle 3452\rangle W . W-6\langle W 23\rangle(\langle W 51\rangle\langle 2345\rangle+\langle W 25\rangle\langle 1345\rangle)] \\
& +\langle\star 24\rangle[\langle 1235\rangle\langle 3451\rangle W . W-6\langle W 51\rangle(\langle W 23\rangle\langle 1345\rangle+\langle W 31\rangle\langle 2345\rangle)] \\
& +\langle\star 25\rangle[-\langle 1234\rangle\langle 4513\rangle W . W-6\langle W 34\rangle(\langle W 12\rangle\langle 3451\rangle+\langle W 31\rangle\langle 2451\rangle)] \\
& +\langle\star 35\rangle[\langle 2341\rangle\langle 4512\rangle W . W-6\langle W 12\rangle(\langle W 34\rangle\langle 2451\rangle+\langle W 42\rangle\langle 3451\rangle)] .
\end{aligned}
$$

Plugging in $W$, this evaluates to eq. (6.5).

Open Access. This article is distributed under the terms of the Creative Commons Attribution License (CC-BY 4.0), which permits any use, distribution and reproduction in any medium, provided the original author(s) and source are credited.

\section{References}

[1] N. Arkani-Hamed and E.Y. Yuan, One-Loop Integrals from Spherical Projections of Planes and Quadrics, arXiv:1712.09991 [INSPIRE].

[2] D. Simmons-Duffin, Projectors, Shadows and Conformal Blocks, JHEP 04 (2014) 146 [arXiv: 1204.3894] [INSPIRE].

[3] C. Bogner and S. Weinzierl, Feynman graph polynomials, Int. J. Mod. Phys. A 25 (2010) 2585 [arXiv: 1002.3458] [INSPIRE].

[4] C. Itzykson and J.B. Zuber, Quantum Field Theory, in International Series In Pure and Applied Physics, McGraw-Hill, New York U.S.A. (1980).

[5] V.A. Smirnov, Evaluating Feynman integrals, Springer Tracts Mod. Phys. 211 (2004) 1 [INSPIRE].

[6] V.A. Smirnov, Analytic tools for Feynman integrals, Springer Tracts Mod. Phys. 250 (2012) 1 [INSPIRE].

[7] H. Cheng and T.T. Wu, Expanding Protons: Scattering at High-Energies, MIT Press, Cambridge Massachusetts U.S.A. (1987) [INSPIRE].

[8] A. Hodges, Eliminating spurious poles from gauge-theoretic amplitudes, JHEP 05 (2013) 135 [arXiv:0905.1473] [INSPIRE].

[9] N. Arkani-Hamed, J.L. Bourjaily, F. Cachazo and J. Trnka, Local Integrals for Planar Scattering Amplitudes, JHEP 06 (2012) 125 [arXiv: 1012.6032] [INSPIRE].

[10] H. Hannesdottir and M.D. Schwartz, S-Matrix for massless particles, Phys. Rev. D 101 (2020) 105001 [arXiv: 1911.06821] [INSPIRE].

[11] H. Hannesdottir and M.D. Schwartz, A Finite S-matrix, arXiv:1906.03271 [INSPIRE].

[12] L.J. Dixon, Gluon scattering in $N=4$ super-Yang-Mills theory from weak to strong coupling, PoS RAD COR2007 (2007) 056 [arXiv:0803.2475] [INSPIRE]. 
[13] L. Magnea, All-order results for soft and collinear gluons, Pramana 72 (2009) 69 arXiv:0806.3353 [INSPIRE].

[14] G.F. Sterman, Partons, factorization and resummation, TASI 95, in proceedings of the Theoretical Advanced Study Institute in Elementary Particle Physics (TASI 95): QCD and Beyond, Boulder, CO, U.S.A., 4-30 June 1995, pp. 327-408 [hep-ph/9606312] [INSPIRE].

[15] E. Gardi and L. Magnea, Infrared singularities in QCD amplitudes, Frascati Phys. Ser. 50 (2010) 137 [arXiv:0908.3273] [INSPIRE].

[16] Z. Bern, L.J. Dixon and V.A. Smirnov, Iteration of planar amplitudes in maximally supersymmetric Yang-Mills theory at three loops and beyond, Phys. Rev. D 72 (2005) 085001 [hep-th/0505205] [INSPIRE].

[17] N. Beisert, B. Eden and M. Staudacher, Transcendentality and Crossing, J. Stat. Mech. 0701 (2007) P01021 [hep-th/0610251] [INSPIRE].

[18] Z. Bern, M. Czakon, L.J. Dixon, D.A. Kosower and V.A. Smirnov, The Four-Loop Planar Amplitude and Cusp Anomalous Dimension in Maximally Supersymmetric Yang-Mills Theory, Phys. Rev. D 75 (2007) 085010 [hep-th/0610248] [INSPIRE].

[19] R.H. Boels, T. Huber and G. Yang, The nonplanar cusp and collinear anomalous dimension at four loops in $\mathcal{N}=4$ SYM theory, PoS RAD COR2017 (2017) 042 [arXiv:1712.07563] [INSPIRE].

[20] R.H. Boels, T. Huber and G. Yang, The Sudakov form factor at four loops in maximal super Yang-Mills theory, JHEP 01 (2018) 153 [arXiv:1711.08449] [INSPIRE].

[21] R.H. Boels, T. Huber and G. Yang, Four-Loop Nonplanar Cusp Anomalous Dimension in $N=4$ Supersymmetric Yang-Mills Theory, Phys. Rev. Lett. 119 (2017) 201601 [arXiv: 1705.03444] [INSPIRE].

[22] G.P. Korchemsky and A.V. Radyushkin, Loop Space Formalism and Renormalization Group for the Infrared Asymptotics of QCD, Phys. Lett. B 171 (1986) 459 [INSPIRE].

[23] G.P. Korchemsky and G. Marchesini, Resummation of large infrared corrections using Wilson loops, Phys. Lett. B 313 (1993) 433 [INSPIRE].

[24] Z. Bern, L.J. Dixon and D.A. Kosower, Dimensionally regulated pentagon integrals, Nucl. Phys. B 412 (1994) 751 [hep-ph/9306240] [INSPIRE].

[25] H. Elvang and Y.-t. Huang, Scattering Amplitudes in Gauge Theory and Gravity, Cambridge University Press (2015).

[26] N. Arkani-Hamed, F. Cachazo, C. Cheung and J. Kaplan, The S-matrix in Twistor Space, JHEP 03 (2010) 110 [arXiv:0903.2110] [INSPIRE].

[27] F. Cachazo, Sharpening The Leading Singularity, arXiv:0803.1988 [InSPIRE].

[28] E.I. Buchbinder and F. Cachazo, Two-loop amplitudes of gluons and octa-cuts in $N=4$ super Yang-Mills, JHEP 11 (2005) 036 [hep-th/0506126] [INSPIRE].

[29] Z. Bern, L.J. Dixon and D.A. Kosower, Dimensionally regulated one loop integrals, Phys. Lett. B 302 (1993) 299 [Erratum ibid. 318 (1993) 649] [hep-ph/9212308] [INSPIRE].

[30] J.L. Bourjaily, S. Caron-Huot and J. Trnka, Dual-Conformal Regularization of Infrared Loop Divergences and the Chiral Box Expansion, JHEP 01 (2015) 001 [arXiv:1303.4734] [INSPIRE]. 
[31] N. Arkani-Hamed, A. Hodges and J. Trnka, Positive Amplitudes In The Amplituhedron, JHEP 08 (2015) 030 [arXiv: 1412.8478] [INSPIRE].

[32] J.L. Bourjaily, F. Dulat and E. Panzer, Manifestly Dual-Conformal Loop Integration, Nucl. Phys. B 942 (2019) 251 [arXiv:1901.02887] [InSPIRE].

[33] S. Abreu, R. Britto, C. Duhr and E. Gardi, Algebraic Structure of Cut Feynman Integrals and the Diagrammatic Coaction, Phys. Rev. Lett. 119 (2017) 051601 [arXiv:1703.05064] [INSPIRE].

[34] S. Abreu, R. Britto, C. Duhr and E. Gardi, Cuts from residues: the one-loop case, JHEP 06 (2017) 114 [arXiv: 1702.03163] [INSPIRE].

[35] L.J. Dixon, M. von Hippel, A.J. McLeod and J. Trnka, Multi-loop positivity of the planar $\mathcal{N}=4$ SYM six-point amplitude, JHEP 02 (2017) 112 [arXiv: 1611.08325] [INSPIRE].

[36] N. Arkani-Hamed, J.L. Bourjaily, F. Cachazo, A.B. Goncharov, A. Postnikov and J. Trnka, Grassmannian Geometry of Scattering Amplitudes, Cambridge University Press (2016) [arXiv: 1212.5605] [INSPIRE].

[37] N. Arkani-Hamed and J. Trnka, The Amplituhedron, JHEP 10 (2014) 030 [arXiv: 1312.2007] [INSPIRE].

[38] N. Arkani-Hamed, Y. Bai and T. Lam, Positive Geometries and Canonical Forms, JHEP 11 (2017) 039 [arXiv: 1703.04541] [INSPIRE]. 